\title{
Saccade Target Selection Relies on Feedback Competitive Signal Integration
}

\author{
Joke P. Kalisvaart, ${ }^{1}$ André J. Noest, ${ }^{2}$ Albert V. van den Berg, ${ }^{1}$ and Jeroen Goossens ${ }^{1}$ \\ ${ }^{1}$ Radboud University Nijmegen Medical Centre, Donders Institute for Brain, Cognition and Behaviour, Department of Cognitive Neuroscience, Section \\ Biophysics, 6500 HB Nijmegen, The Netherlands, and ²Developmental Biology Department, Utrecht University, 3584 CH Utrecht, The Netherlands
}

It is often assumed that decision making involves neural competition, accumulation of evidence "scores" over time, and commitment to a particular alternative once its scores reach a critical decision threshold first. So far, however, neither the first-to-threshold rule nor the nature of competition (feedforward or feedback inhibition) has been revealed by experiments. Here, we presented two simultaneously flashed targets that reversed their intensity difference during each presentation and instructed human subjects to make a saccade toward the brightest target. All subjects preferentially chose the target that was brightest during the first stimulus phase. Unless this first phase lasted only $40 \mathrm{~ms}$, this primacy effect persisted even if the second, reversed-intensity phase lasted longer. This effect did not result from premature commitment to the initially dominant target, because a strong target imbalance in the opposite direction later drove nearly all responses toward that location. Moreover, there was a nonmonotonic relation between target imbalance and primacy: increasing the target imbalance beyond $40 \mathrm{~cd} / \mathrm{m}^{2}$ caused an attenuation of primacy. These are the hallmarks of hysteresis, predicted by models in which target representations compete through strong feedback. Reaction times were independent of the choice probability. This dissociation suggests that target selection and movement initiation are distinct phenomena.

\section{Introduction}

To explain how saccadic responses compete for selection and execution, current decision-making theories assume that sensory evidence is noisy and that it is accumulated over time to reach a decision bound (Ratcliff and McKoon, 2008). Different types of models make different assumptions about how evidence is combined (Smith and Ratcliff, 2004): "evidence scores" for two or more alternatives can accumulate independently (Cousineau, 2004), compete with feedforward inhibitory interaction (Laming, 1966; Link and Heath, 1975; Nosofsky and Palmeri, 1997; Palmeri, 1997), or compete via feedback inhibition (Usher and McClelland, 2001; Wang, 2002; Wong and Wang, 2006). So far, it has been impossible to single out one among the many types of visual decision-making models; despite different architectures, they predict similarly optimal choice behavior in static twoalternative forced choice tasks (model mimicry; van Zandt and Ratcliff, 1995; Bogacz et al., 2006).

To distinguish between feedforward and feedback crossinhibition, we introduced a new manipulation: we studied saccadic choices between pairs of briefly flashed targets the intensities

\footnotetext{
Received May 30, 2012; revised June 7, 2013; accepted June 18, 2013.

Author contributions: A.J.N., A.V.v.d.B., and J.G. designed research; J.P.K. performed research; J.P.K. and J.G. analyzed data; J.P.K., A.J.N., A.V.v.d.B., and J.G. wrote the paper.

This work was supported by the The Netherlands Organisation for Scientific Research (Grant \#864.06.005 ALW VIDI to J.G.) and the Radboud University Nijmegen Medical Centre. We thank M. van Beek, V. Gonzales, and J. Roelofs for their contributions to the experimental work.

The authors declare no competing financial interests.

Correspondence should be addressed to Jeroen Goossens, Radboud University Nijmegen Medical Centre, Donders Institute for Brain, Cognition and Behaviour, Department of Cognitive Neuroscience, section Biophysics, P.O. Box 9101, 6500 HB Nijmegen, The Netherlands. E-mail: j.goossens@donders.ru.nl.

DOI:10.1523/JNEUROSCI.2613-12.2013

Copyright $\odot 2013$ the authors $\quad 0270-6474 / 13 / 3312077-13 \$ 15.00 / 0$
}

of which were swapped during presentation (Fig. $1 A, B$ ). Under such conditions, circuits with feedback competition that exceed a certain critical strength are sensitive to the initial stimulus bias but relatively resistant to the later bias reversal. This characteristic behavior (hysteresis) occurs because the initial "winner" maintains its advantage by recurrent inhibition of its competitor (Noest et al., 2007; Furman and Wang, 2008). These models thus predict that subjects typically choose the target with the highest initial strength provided that the initial differences outlast the time constant of the feedback creating the hysteresis and the stimuli are not strong enough to overcome this hysteresis (Fig. 1C). In contrast, feedforward integration models predict that for balanced durations of the two stimulus epochs, early and late biases are equally effective (because the total evidence for both targets is the same), whereas leaky-integrator models even predict that later biases dominate the final evidence scores. This implies that subjects should either show no preference at all or show a preference for the target that is strongest at the end, unless they make an irreversible choice for the initially strongest target before the stimulus ends (premature choice commitment). Indeed, all accumulator models can produce primacy (i.e., a choice preference for the initially strongest target) if they include some thresholding mechanism that induce choice commitment. We probed the contribution of such thresholding mechanisms by appending strong intensity biases toward the initially weakest target.

We found robust preferences for the initially strongest target, which reversed only if the first stimulus epoch was sufficiently short and the second epoch outlasted the first one by a sufficient amount. The basic primacy effect was attenuated at larger stimulus biases and could even be inverted completely if the stimuli were immediately followed by a strong intensity bias toward the 


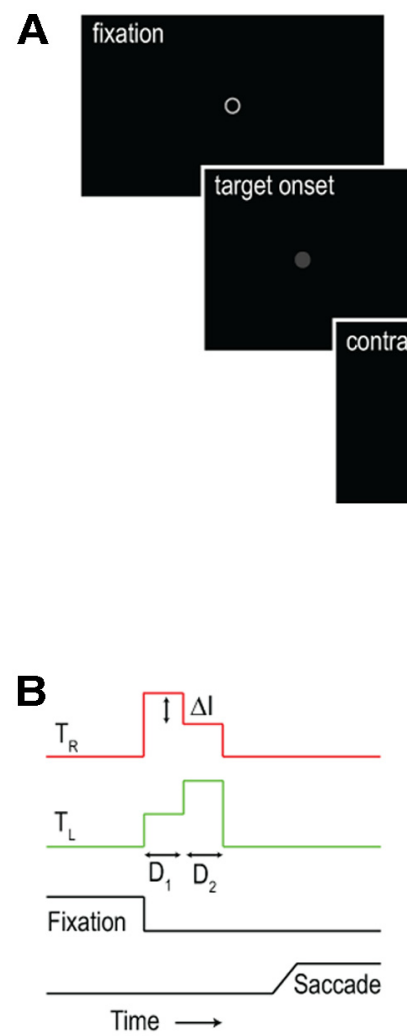

Figure 1. Intensity reversal paradigm and model predictions. $A$, In each trial, a central fixation ring was presented for $400-1200 \mathrm{~ms}$. Upon disappearance of the fixation ring, two peripheral targets with different intensities $(\Delta /)$ appeared. After a delay of $40-120 \mathrm{~ms}\left(D_{1}\right)$, the intensities of the left and right target reversed (reversal trials) or remained unaltered (control trials, data not shown). In either case, both targets remained present for another $40-120 \mathrm{~ms}$ $\left(D_{2}\right)$ until they were extinguished simultaneously. Subjects were instructed to look at the fixation ring and make a saccade toward the most intense target as quickly and as accurately as possible. $\boldsymbol{B}$, Time traces illustrating the intensity changes of the right target $\left(T_{\mathrm{R}}\right)$, the left target $\left(T_{\perp}\right)$, and the fixation ring, as well as the ensuing saccade. Depicted traces show an initial intensity bias toward the right target, followed by an equally strong bias toward the left target. The intensity differences between the left and right target, the timing of the intensity reversals, and the overall stimulus durations were systematically varied. In a modified version of this paradigm, the $D_{1}$ and $D_{2}$ epochs were followed by a third epoch $\left(D_{3}\right)$ in which there was a strong intensity bias toward the initially weakest target. See Materials and Methods for details. $C$, Response of competing decision units. Models with sufficiently strong feedback competition predict a choice preference for targets with highest initial strength. Feedforward integration models predict equal choice probabilities because the early and late biases are equally effective. Feedforward models with leaky integration predict that later biases dominate, resulting in a choice preference for targets with highest final strength. Premature commitment induced by low decision thresholds (dashed horizontal lines) predicts primacy for any of these architectures, but in such bounded accumulator models, primacy cannot be undone by later stimuli.

initially weakest target. This near-complete transition from primacy to recency shows that the observed primacy cannot be attributed to premature commitment to the initially strongest target. Simulations showed that all these results were best described by a feedback cross-inhibition model. Reaction times were independent of the changes in choice probability and instead depended on the initial intensity of the selected target.

\section{Materials and Methods}

Subjects

Ten adult human subjects ( 5 male, 5 female) participated in the experiments. All had normal or corrected-to-normal visual acuity. Subjects were informed about the experimental procedures and gave informed consent before the start of the experiments. Procedures were approved by the Radboud University Medical Centre. Subjects J.R., V.G., J.G., and J.K. were experimenters; all other subjects were kept unaware of the aim of the study.

\section{Setup}

Subjects were seated in a darkened room at $80 \mathrm{~cm}$ of a projection screen on which stimuli were back projected. In the first three experiments, an LCD projector (model DLA-S10E; JVC, ) with a refresh rate of $75 \mathrm{~Hz}$ and a maximum luminance level of $\sim 45 \mathrm{~cd} / \mathrm{m}^{2}$ was used. To present targets at higher contrast and luminance levels in Experiment 4, we used a digital light processing projector with a maximum luminance level of $\sim 300$ $\mathrm{cd} / \mathrm{m}^{2}$ and a refresh rate of $60 \mathrm{~Hz}$ (P1265 model DNX0702; Acer). Luminance levels were measured with a luminance meter (model LS-100; Minolta).

A chinrest was used to minimize head movements. Eye movements were measured with the scleral search coil technique (Remmel Laboratories). Coils were inserted after one drop of topical anesthetic (oxybuprocaine hydochloride $0.4 \%$; Thea Pharma). Once the coil was in place, a drop of artificial tear (methylcellulose $0.5 \%$; Thea Pharma) and a bandage lens (a large contact lens with a strength of zero diopters) were applied to minimize ocular discomfort. Use of the bandage lens doubled the measuring time with the coil to $\sim 1 \mathrm{~h}$ per session (Sprenger et al., 2008). Eye position signals were low-pass filtered, amplified, and sampled at $500 \mathrm{~Hz}$ per channel. The spatial resolution of the horizontal and vertical eye position signals was better than $0.1^{\circ}$ (root mean square measure).

\section{Paradigms}

In the first three experiments, each trial consisted of four epochs (Fig. 1). At the beginning of each trial, a fixation ring with diameter of $0.5^{\circ}$ was presented at the center of the screen. Then, after a random period of $400-1200 \mathrm{~ms}$, the fixation ring disappeared and two filled, circular targets with a diameter of $0.5^{\circ}$ were presented simultaneously at $10^{\circ}$ to the left and right of the center. After a variable delay $\left(D_{1}\right)$ of 40,80 , or $120 \mathrm{~ms}$, this first set of targets was replaced by a second set of targets at the same locations. In control trials, the intensity of both the left and right target remained unaltered. In reversal trials, however, the intensities of the left and right target were reversed compared with the first target epoch. In both cases, targets were then displayed for another 40,80 , or $120 \mathrm{~ms}\left(D_{2}\right)$ until the screen turned black. For each reversal condition, control stimuli were presented with the same total duration (i.e., $D_{1}+D_{2}$ ).

Subjects were always instructed to first look at the fixation ring and then make a saccade to the most intense target as quickly and accurately as possible. They received no feedback about their performance. Target intensities and durations of the first and second target presentation epoch were manipulated systematically across trials and experimental sessions.

Target intensities in Experiments 1-3 ranged from 4.5 to $36.6 \mathrm{~cd} / \mathrm{m}^{2}$. Background luminance was $0.273 \mathrm{~cd} / \mathrm{m}^{2}$. The intensity difference $(\Delta I)$ between the left and the right target on any given trial could be large ( 4.5 vs $\left.36.6 \mathrm{~cd} / \mathrm{m}^{2}\right)$, medium $\left(7.8 \mathrm{vs} 29.1 \mathrm{~cd} / \mathrm{m}^{2}\right)$, small ( 12.3 vs $\left.22.4 \mathrm{~cd} / \mathrm{m}^{2}\right)$, or zero (both targets $16.7 \mathrm{~cd} / \mathrm{m}^{2}$ ). Thus, in control trials, target luminance could be unambiguous or ambiguous. In reversal trials, the mean intensity of the left and right target was the same only when the durations of the first and second epoch were the same. High-to-low and low-tohigh intensity changes were achieved with a time constant of $\sim 10 \mathrm{~ms}$, limiting the shortest practical presentation time to $40 \mathrm{~ms}$.

Experiments 1-3 established the conditions under which primacy arises, but could not discriminate between two very different mechanisms that can generate primacy: hysteresis due to strong feedback competition or premature commitment due to an absorbing bound (see Introduction). Testing whether primacy can still be reverted does discriminate between these different model types. Primacy due to absorbing bounds is by definition irreversible and should increase monotonically with increasing $\Delta I$. Conversely, primacy due to hysteretic integration dynamics should be reversible by a sufficiently strong stimulus. In Experiment 4 , we therefore presented reversal stimuli across an extended range of target contrasts (i.e., from $22.5 \mathrm{vs} 33.6 \mathrm{~cd} / \mathrm{m}^{2}$ up to $1.5 \mathrm{vs} 77.5 \mathrm{~cd} / \mathrm{m}^{2}$ ). The durations $D_{1}$ and $D_{2}$ were kept fixed at $50 \mathrm{~ms}$. Targets in the $100 \mathrm{~ms}$ 
ambiguous condition had an intensity of $27.8 \mathrm{~cd} / \mathrm{m}^{2}$. Control stimuli lasted $50 \mathrm{~ms}$ and target contrasts ranged from 7.2 versus $49.5 \mathrm{~cd} / \mathrm{m}^{2}$ to 26.0 versus $29.7 \mathrm{~cd} / \mathrm{m}^{2}$. Background luminance was $0.18 \mathrm{~cd} / \mathrm{m}^{2}$.

To further probe the extent to which primacy can be undone by later stimulus evidence, Experiment 4 also included two forced reversal conditions in which the stimulus presentation ended with a bright pulse at the location of the initially weakest target. More specifically, in the 50-50-67 ms forced reversal condition, a 50-50 ms reversal stimulus was immediately followed by a third, $67 \mathrm{~ms}$ epoch in which there was a strong bias toward the initially weakest target. In the 83-67 ms forced reversal condition, $83 \mathrm{~ms}$ of initial bias was immediately followed by $67 \mathrm{~ms}$ of reversed extreme bias. In both cases, the initial bias was 49.5 versus 7.2 $\mathrm{cd} / \mathrm{m}^{2}$ and the final, reversed bias was 7.2 versus $283 \mathrm{~cd} / \mathrm{m}^{2}$. For subjects J.K. and J.G., we also tested forced reversal conditions with an initial bias of 13.7 versus $40.0 \mathrm{~cd} / \mathrm{m}^{2}$ and 3.6 versus $62.7 \mathrm{~cd} / \mathrm{m}^{2}$, respectively, because those intensity differences better matched the intensity differences that evoked the strongest primacy effect in the unforced condition. As in Experiments 1-3, we kept the stimulus durations short to ensure that the subjects would not consciously perceive the intensity manipulations.

Data were collected in blocks of 120-138 trials in which all experimental conditions were presented five or six times in pseudorandom order. Subjects completed 10-12 blocks across different sessions until each unique condition was tested 60 times (Experiments 1-3) or at least 100 times (Experiment 4). This typically required three or four sessions per subject per experiment.

\section{Data analysis}

Saccades were detected on the basis of calibrated eye position signals with custom software. Detection of saccade onsets and offsets was based on velocity and acceleration criteria. All saccade markers were examined by the experimenters and, if necessary, corrected. Further analysis was done with MATLAB (version 7.9, MathWorks) using custom software.

To determine the subject's choice regarding the most intense target, we only considered the direction of his/her first saccadic eye movement after stimulus offset that exceeded the detection criteria of $0.5^{\circ}$ amplitude and $30 \%$ velocity. Subsequent correction saccades in the opposite direction were discarded. Such corrections occurred only in $\sim 1.5 \%$ of trials with equal probability across all stimulus conditions. Therefore, excluding trials in which corrections occurred did not change any of our conclusions.

Psychometric response functions. A generalized linear model with a logit link function was used to fit psychometric curves through the data points from Experiments 1-3 using the following logistic equation:

$$
P_{R}=\frac{1}{1+e^{-Q}} \text { with } Q=a \cdot \Delta I+b
$$

Where $P_{R}$ is the probability of a rightward saccade and $\Delta I$ the intensity difference (in $\mathrm{cd} / \mathrm{m}^{2}$ ) between the right and the left target at stimulus onset. Positive values of $\Delta I$ indicate that the (initial) intensity of the right target is larger than that of the left target. The parameters $a$ and $b$ represent the subject's sensitivity and bias, respectively. Student's $t$ tests were used to test whether the fit parameters significantly differed from zero and whether the slopes $(a)$ of the psychometric curves differed between experimental conditions.

The simple logistic model from Equation 1 was no longer adequate to describe the nonmonotonic psychometric curves obtained in Experiment 4 . We therefore extended the model in the following way:

$$
P_{R}=\frac{1}{1+e^{-Q}} \text { with } Q=d+a \cdot(\Delta I-b)+c \cdot(\Delta I-b)^{3}
$$

Note that $Q$ is now a third-order polynomial where the additional parameter $c$ represents the strength of the hysteresis reductions at higher $\Delta I$ 's (see Results). The parameters $b$ and $d$ capture the subject-specific response biases. The horizontal shift $b$ reflects an offset in the input to the choice stage. The vertical shift $d$ reflects some bias at the output of that stage. Experiment 4 brings out the distinction between these two types of biases. In Experiments 1-3, they are confounded into one net bias pa- rameter (i.e., $b$ in Equation 1) because the applied range of target contrasts appeared to be too small in these experiments.

Absorbing bounds inequality test. To quantify how strongly the data from Experiment 4 challenge mechanisms that attribute our primacy effects to premature commitment caused by absorbing bounds, we consider the class of accumulator models that have absorbing bounds but no hysteresis in their evidence accumulation. This whole class of models can then be tested by comparing the forced reversal conditions with their unforced counterparts. Key in this analysis is the following notion: at the end of $D_{2}$ (which is either the end of the whole stimulus or the start of forcing $D_{3}$ phase) a fraction of the accumulated-evidence trajectories already reached the boundary of the initially strongest target (unknown fraction $A_{i}$ ) or the boundary for the other target (unknown fraction $A_{f}$ ), leaving only a limited fraction $\left(1-A_{i}-A_{f}\right)$ of formally "undecided" trajectories that can still be influenced by a later forcing stimulus (Fig. $5 A$ ). If we knew for sure that the bright $D_{3}$ forcing pulse were strong enough to drive all "surviving" trajectories to (or closest to) the boundary of the initially weakest target, the probability of still choosing the initially strongest target in the forced reversal condition would provide a direct measure of $A_{i}$. Unfortunately, we cannot be sure that the forcing stimulus is indeed sufficiently strong to achieve this. We can, however, show that the paired probabilities from the two experimental conditions must satisfy an inequality that - if violated by our data—rejects this whole class of bounded accumulator models:

Let $F_{i}$ denote the probability of still choosing the initially strongest target in the forced reversal condition. This observed probability then sets the upper limit for the unknown fraction of trials, $A_{i}$, in which the accumulated-evidence trajectories reached the absorbing bound of the initially strongest target before the end of $D_{2}$. Note that we can only conclude that $F_{i} \geq A_{i}$, because, as we noted above, we cannot be sure that the $D_{3}$ forcing stimulus is strong enough. However, from the time- and polarity-antisymmetric nature of the reversal stimulus and from the observed behavior in the (unforced) reversal condition, we can now derive further constraints on $A_{i}$.

For simplicity, we first assume absence of response biases. This assumption is eventually lifted, because it can be shown to only make our test more conservative. Now let $R_{i}$ denote the observed probability of choosing the initially dominant target in the (unforced) reversal condition. Next, keep in mind that $R_{i}$ is the sum of $A_{i}$ and the probability that an undecided trajectory ends up being closer to the bound of the initially strongest target (Fig. 5A). Next, we know that $A_{i} \geq A_{f}$. This holds because at the end of $D_{1}$, the trajectories tend to be closer to the boundary of the initially brightest target (as verified by the control condition). Therefore, given that $D_{2}=D_{1}$ and given the same (but reversed) $\Delta I$, a drift to the other boundary during $D_{2}$ takes more time. This, in combination with the assumed lack of hysteresis and bias in this model class, implies that at most one half of the undecided trajectories can eventually contribute to choosing the initially strongest target. That gives us the starting point for some algebra which relates $R_{i}$ to $F_{i}$ :

$$
R_{i} \leq A_{i}+\frac{1}{2}\left(1-A_{i}-A_{f}\right)=\frac{1}{2}\left(1+A_{i}-A_{f}\right) \leq \frac{1}{2}\left(1+F_{i}\right)
$$

or:

$$
F_{i} \geq 2 R_{i}-1
$$

Therefore, we can test whether the measured $R_{i}$ and $F_{i}$ combinations violate this inequality.

The only remaining step required for the inequality test of Equation 4 is to account for the effect of choice biases that were clearly present in our data. We can do this by averaging the "raw" $R_{i}$ and $F_{i}$ values plotted in Figure 4 for the two mirror-symmetric stimulus conditions per subject. This results in more bias-resistant measures of $R_{i}$ and $F_{i}$ :

$$
\hat{R}_{i}=\frac{1}{2}\left(R_{i}^{+}+1-R_{i}^{-}\right) \text {and } \hat{F}_{i}=\frac{1}{2}\left(F_{i}^{+}+1-F_{i}^{-}\right)
$$

Where the + and - superscripts refer to initial stimulus biases of $+\Delta I$ and $-\Delta I$, respectively. Using the modified $R_{i}$ and $F_{i}$ variables in this test 
not only reduces the effect of subject-dependent biases (which are difficult to estimate precisely), but the remaining "second-order" bias effects only makes our test more conservative (at least in a wide regime of practical interest, $\hat{F}_{i}<0.5$, because of convexity properties of the psychometric functions).

SEs for $\hat{R}_{i}$ and $\hat{F}_{i}$ were obtained from error-propagation rules:

$$
\begin{aligned}
& S E\left(\hat{R}_{i}\right)=\sqrt{\frac{R_{i}^{+}\left(1-R_{i}^{+}\right)}{4 N_{R}^{+}}+\frac{R_{i}^{-}\left(1-R_{i}^{-}\right)}{4 N_{R}^{-}}} \text {and } \\
& \qquad S E(\hat{F})_{i}=\sqrt{\frac{F_{i}^{+}\left(1-F_{i}^{+}\right)}{4 N_{F}^{+}}+\frac{F_{i}^{-}\left(1-F_{i}^{-}\right)}{4 N_{F}^{-}}}
\end{aligned}
$$

Where the Ns denote the total numbers of trials per test condition, with the subscripts $\mathrm{R}$ and $\mathrm{F}$ referring to the reversal conditions and forced reversal conditions, respectively, and with the + and - superscripts referring to the two different intensity biases as before. Using normal approximation, we then tested the null hypothesis from Equation 4 against the alternative hypothesis (i.e., $F_{i}<2 R_{i}-1$ ) by applying the following one-sided $z$ test:

$$
z=\frac{2 \hat{R}_{i}-1-\hat{F}_{i}}{S E} \text { with } S E=\sqrt{4 \cdot S E\left(\hat{R}_{i}\right)^{2}+S E\left(\hat{F}_{i}\right)^{2}}
$$

Chronometric response functions. Saccade latencies were also analyzed as a function of $\Delta I$. For each subject, we first subtracted the average reaction time measured in the ambiguous control condition pooled across rightward and leftward saccades. This was done because the average latency relative to target onset differed greatly among subjects (range: 250-600 $\mathrm{ms}$ ). The resulting chronometric response functions were quantified by fitting linear regression lines to the data:

$$
\Delta R T=\alpha \cdot \Delta I+\beta
$$

One-way ANCOVA, with $\Delta I$ as the predictor and $\Delta R T$ (in ms) as the response and condition as the grouping variable, was used to test for differences between the chronometric curves. Correction for multiple testing was achieved by using a Tukey-Kramer test for multiple comparisons.

\section{Results}

\section{Balanced durations}

In the first experiment, two targets were presented simultaneously for 80 or $160 \mathrm{~ms}$, and the initial intensity bias reversed halfway during stimulus presentation (i.e., $D_{1}=D_{2}=40 \mathrm{~ms}$ or $D_{1}=D_{2}=80 \mathrm{~ms}$ ). In control trials, the intensities remained the same across $D_{1}$ and $D_{2}$. Figure 2 shows the probability of rightward saccades as a function of the initial rightward intensity bias ( $\Delta I$, positive if the right target was brightest at stimulus onset). In both control conditions (Fig. $2 A, C$ ), subjects discriminated target intensities easily; the psychometric curves had steep, positive slopes and saccades were almost always directed toward the brightest target at the largest intensity differences $(\Delta I= \pm 36$ $\left.\mathrm{cd} / \mathrm{m}^{2}\right)$. For purely ambiguous control stimuli $\left(\Delta I=0 \mathrm{~cd} / \mathrm{m}^{2}\right)$, choice probabilities scattered around $50 \%$. The psychometric curves obtained in the $80-80 \mathrm{~ms}$ reversal condition (Fig. $2 \mathrm{~B}$ ) also had steep, positive slopes, indicating a significant preference for the initially strongest target in all subjects ( $t$ test, $p<0.0001$ ). Subjects even maintained a clear preference for the initially brightest target in the 40-40 ms reversal condition (Fig. 2D; slopes $>0$; $t$ test, $p<0.001$ for all subjects). Compared with the control conditions, the slopes were somewhat reduced (80-80 ms condition: $t$ test, $p<0.05$ for all subjects), or reduced considerably (40-40 ms reversal condition: $t$ test, $p<0.01$ for all subjects except V.G.), indicating that target selection was not exclusively based on the initial intensity differences; subsequent stimulus information also had a significant influence on the eventual de-
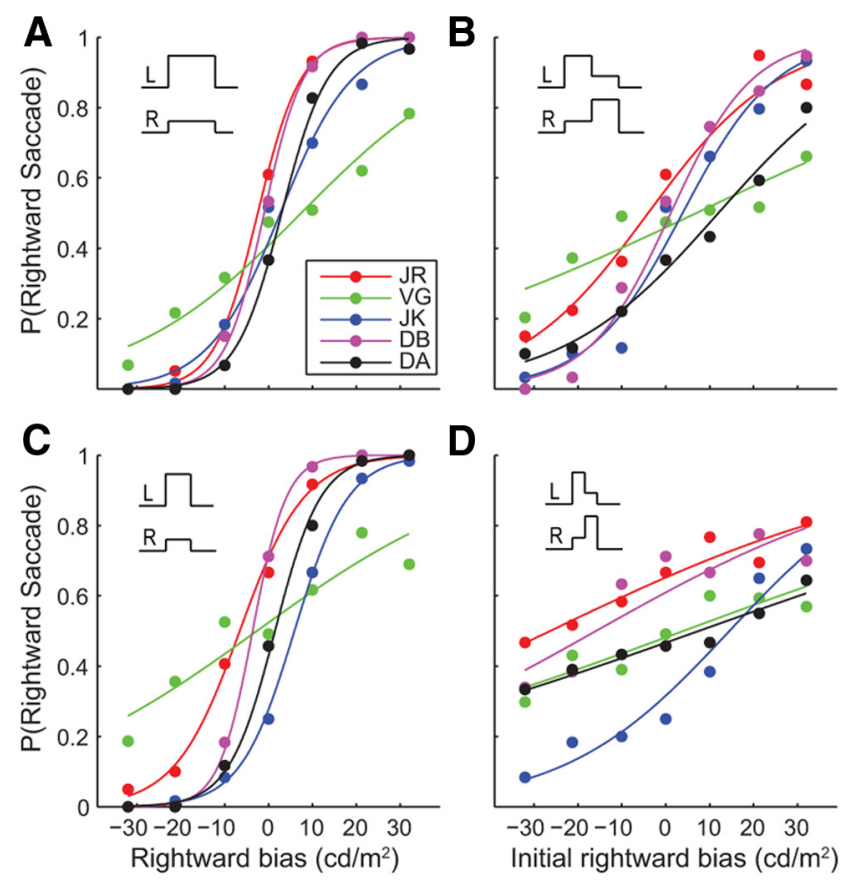

Figure 2. Initially brightest target was preferred despite balanced target durations. Shown is the probability of rightward saccades as a function of the (initial) intensity difference between the right and left target in Experiment 1. A, The $160 \mathrm{~ms}$ control trials. $\boldsymbol{B}$, The $80-80 \mathrm{~ms}$ reversal trials. $\boldsymbol{C}$, The $80 \mathrm{~ms}$ control trials. $\boldsymbol{D}$, The $40-40 \mathrm{~ms}$ reversal trials. Each data point consists of 60 trials. Solid lines show psychometric functions fitted to the data. Colors identify the different subjects. Insets illustrate the intensities of the two targets over the course of a trial. The positive slopes of the psychometric curves indicate a consistent preference for the initially strongest target (compared with the ambiguous control condition). Negative slopes would have been obtained if there had been a preference for the target that had the highest final intensity. Horizontal lines would have been obtained if there had been no preference (other than a constant bias).

cision, even though the changes in target luminance were not consciously perceived.

\section{Unbalanced durations}

To probe further the contributions of initial and later stimulus epochs, two additional experiments were performed in which durations $D_{1}$ and $D_{2}$ were manipulated. In Experiment 2, we first kept the total stimulus duration fixed at $D_{1}+D_{2}=160 \mathrm{~ms}$ and swapped the targets' intensities at $D_{1}=40,80$, or $120 \mathrm{~ms}$ after stimulus onset. Choices in the control condition (Fig. $3 A$ ) and 80-80 ms reversal condition (Fig. $3 C$ ) replicated the primacy effects of Experiment 1 with more subjects. In the new 120-40 ms reversal condition (Fig. $3 B$ ), that is, if $D_{1}$ lasted three times longer than $D_{2}$, choice behavior became almost undistinguishable from the control condition in all subjects. In contrast, if $D_{1}$ was three times shorter than $D_{2}$ in the $40-120$ ms reversal condition, the subjects' choice behaviors diverged considerably (Fig. 3D). Three subjects (I.B., A.M., and J.K.) still showed a significant, albeit weaker primacy effect (slopes $>0$; $t$ test, $p<0.05$ ), but the other four subjects (J.R., V.G., D.B., and D.A.) now showed a significant preference for the target that was most intense at the end (slopes $<0$; $t$ test, $p<0.01$ ). However, even in these four subjects, the choice probabilities were not completely inverted compared with the 120-40 ms condition. To summarize these changes, Figure $3 E$ shows the averaged slopes of the psychometric curves as a function of $\Delta D=D_{1}-D_{2}$. Note the systematic increase in slopes with increasing $\Delta D$. The asymmetric effect of intensity difference 

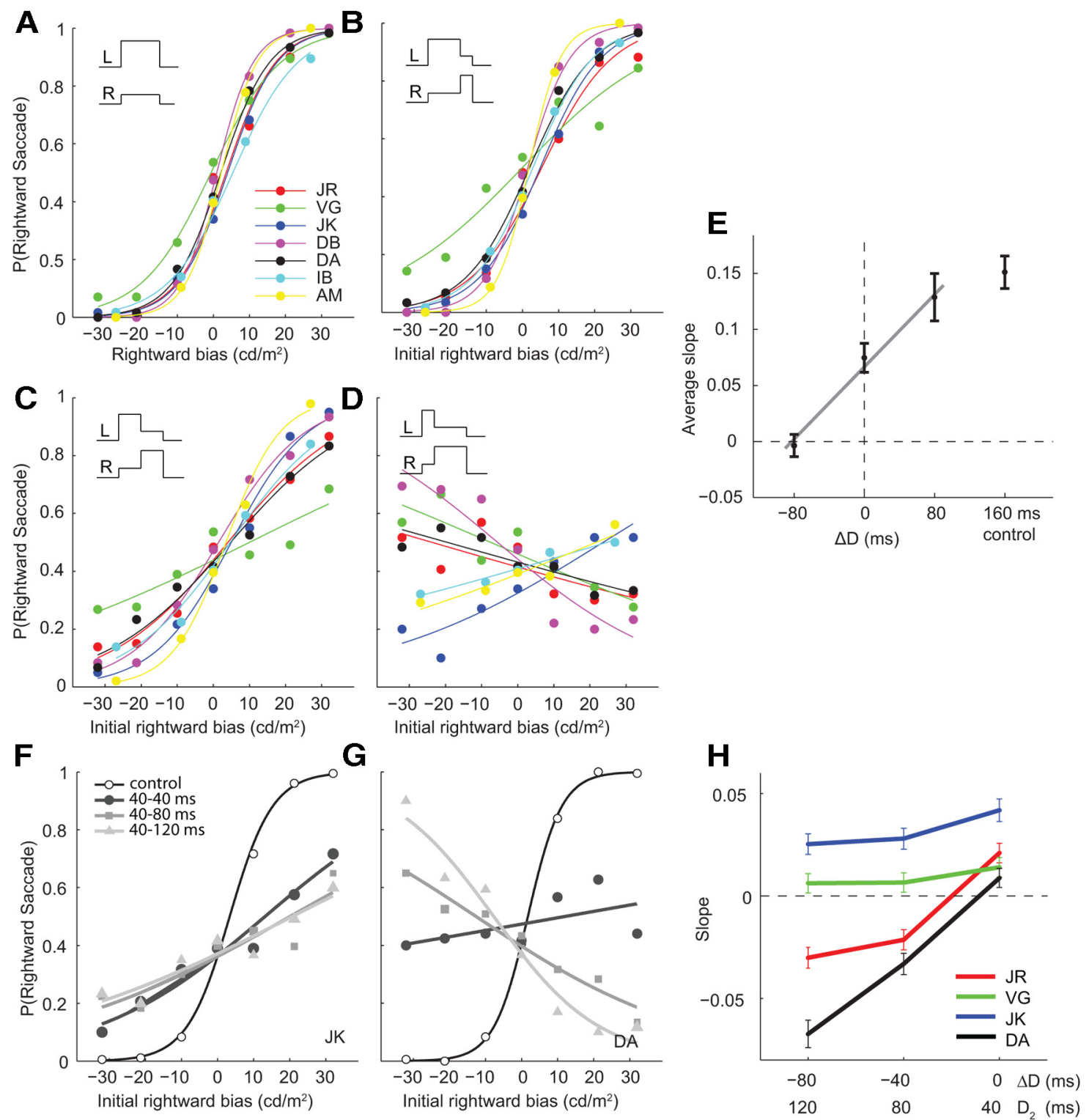

Figure 3. Choice behavior under unbalanced duration conditions. Experiment $2(\boldsymbol{A}-\boldsymbol{E})$ varied $D_{1}$ and $D_{2}$, keeping $D_{1}+D_{2}$ constant at $160 \mathrm{~ms}$. $\boldsymbol{A}$, The $160 \mathrm{~ms}$ control condition. $\boldsymbol{B}$, The $80-80 \mathrm{~ms}$ reversal condition. C, The $120-40 \mathrm{~ms}$ reversal condition. $\boldsymbol{D}$, The $40-120 \mathrm{~ms}$ reversal condition. $\boldsymbol{E}$, Slopes of the psychometric curves for the three different reversal conditions and the $160 \mathrm{~ms}$ control condition as a function of $\Delta D$ (i.e., $D_{1}-D_{2}$ ), averaged over subjects. Experiment $3(\boldsymbol{F}-\boldsymbol{H})$ varied $D_{2}$, keeping $D_{1}$ constant at $40 \mathrm{~ms}$. $\boldsymbol{F}, \boldsymbol{G}$, Psychometric response functions of subjects J.K. and $D$.A., respectively. Black, open circles indicate control data that were pooled over the three target durations $(80,120$, and $160 \mathrm{~ms})$ because stimulus durations in control trials had no significant influence. Dark gray, circles indicate the $40-40 \mathrm{~ms}$ reversal condition. Middle gray, squares indicate the $40-80 \mathrm{~ms}$ reversal condition. Light gray triangles indicate the 40-120 ms reversal condition. $\boldsymbol{H}$, Slopes of the psychometric curves fitted to the three reversal conditions as a function of $\Delta D$. Values of $D_{2}$ are indicated below the horizontal axes for convenience. Positive (negative) slope indicates a preference for the initial (final) brightest target. Colors represent different subjects; error bars denote SEs of the slopes. Note breakdown of the primacy effect if long $D_{2}(\geq 80 \mathrm{~ms}$ ) epochs were preceded by a short $(40 \mathrm{~ms}) D_{1}$ epoch.

in $D_{1}$ versus $D_{2}$ is reflected in the fact that the trend line does not pass through the origin.

Because Experiment 2 kept the total duration constant, one cannot conclude that $\Delta \mathrm{D}$ is the only or even the mechanistically relevant variable controlling the choice process. Indeed, strong hysteresis is only expected if $D_{1}$ exceeds the (probably subjectdependent) feedback time constant. To test this, Experiment 3 used the smallest practical $D_{1}(40 \mathrm{~ms})$ and varied $D_{2}$ among 40 , 80 , and $120 \mathrm{~ms}$ to quantify the potential breakdown of the primacy that we found at larger $D_{1}$. Figure $3 F, G$ illustrates the behavior of two different subjects in this experiment. Note that their choices were consistently influenced by $D_{2}$, with a clear breakdown of primacy at $D_{2} \geq 80 \mathrm{~ms}$ in one of them (Fig. $3 G$ ).
This behavior is quantified in Figure $3 H$ for all four subjects. Note the systematic decrease in slope of the psychometric curves with increasing $D_{2}$ : the longer the $D_{2}$, the weaker the preference for the initially most intense target. For two subjects, the sign of the slopes even flipped from positive to negative if $D_{2}$ exceeded $D_{1}$, indicating a preference for the target with the highest final intensity.

Thus, a brief (40-80 ms) initial bias consistently dominated the responses if $D_{1}$ lasted an equal amount of time or longer than $D_{2}$. However, stimulus information in $D_{2}$ was not simply ignored. In Experiment 2, the longer that $D_{2}$ exceeded $D_{1}$, the more often subjects responded to the target with the highest final intensity and, for the short $D_{1}$ durations in Experiment 3, some but not all subjects showed a transition from primacy to recency. 


\section{Strong target imbalance}

As noted in the Introduction, observing primacy does not necessarily imply that the dynamics of evidence accumulation produces hysteresis. In fact, any decision process that can commit to a particular choice before the stimulus ends can produce primacy independently of its dynamic properties (Fig. 1C). The capability for such premature commitment is common to a wide range of decision models, which share the crucial assumption of becoming committed to a particular choice as soon as the evidence (accumulated via some model-dependent process) for that particular alternative hits a fixed bound or threshold. However, the crucial distinction is that dynamic hysteresis due to feedback cross-inhibition creates primacy without irreversible commitment: sufficiently strong input should be able to "override" hysteresis, even when such input arrives well after the internal dynamics has settled into one of its hysteretic states. We therefore decided to present equal-duration reversal stimuli across an extended range of target imbalances. This yields two clearly different predictions for models with or without strong feedback competition. In the absence of feedback competition or if the feedback is not strong enough to create hysteresis, primacy due to absorbing bounds should increase systematically with target imbalance, so the psychometric curves should increase monotonically from saturation at $p=0$ to saturation at $p=1$. Primacy due to strong feedback competition, however, should produce nonmonotonic curves because primacy will eventually decrease if the target imbalance becomes sufficiently large. This prediction holds because the input during $D_{2}$ will start to exceed the strength of the hysteresis induced during $D_{1}$, and the absence of commitment (absorbing boundaries) will allow the decision process to evolve for at least the full duration of the stimulus. Some of the psychometric curves from Experiments 1-3 may already hint toward such nonmonotonic choice behavior, but testing this prediction over a wider range of target imbalances is clearly called for. For this purpose, we switched to a different projector capable of producing higher contrasts and luminances (see Materials and Methods).

Figure 4 shows the results from all six subjects who participated in this fourth experiment. Dashed curves are the psychometric curve fitted to the control data (Equation 1, circles). For these brief, $50 \mathrm{~ms}$ stimuli, all subjects correctly chose the most intense target in the condition with the highest intensity and correct responses decreased to $\sim 50 \%$ for very small contrast. In the 50-50 ms reversal condition (Equation 1, squares), subjects showed a consistent primacy effect that increased with target imbalance up to $\sim 40 \mathrm{~cd} / \mathrm{m}^{2}$. This regime corresponds to the behavior measured in the 40-40 ms reversal conditions of Experiments 1 and 3. At higher intensity biases, however, the choice probabilities clearly showed a reversed trend, in that the primacy effect started to decline. To determine whether this decline was statistically significant, we first compared the probabilities of the outmost two points on either side of the curve. We found a significant (Fischer's exact test, $p<0.05$ ) decrease in primacy on one side of the range of target contrasts for all subjects except J.G. and a significant decrease on both sides for subject J.E. To further quantify the strength of this trend reversal, we fitted a minimally extended generalized psychometric function model to the entire dataset (Equation 2, solid lines; see Materials and Methods). For all subjects, the coefficient of the third-order polynomial term was significantly negative (mean $\pm \mathrm{SD}$ across subjects: $c=$ $(-2.73 \pm 0.72) \times \cdot 10^{-6}$; $t$ test, $p<0.05$, for all individual subjects), indicating that the data indeed showed a significant decline of the basic primacy effect at higher target contrasts. These tests

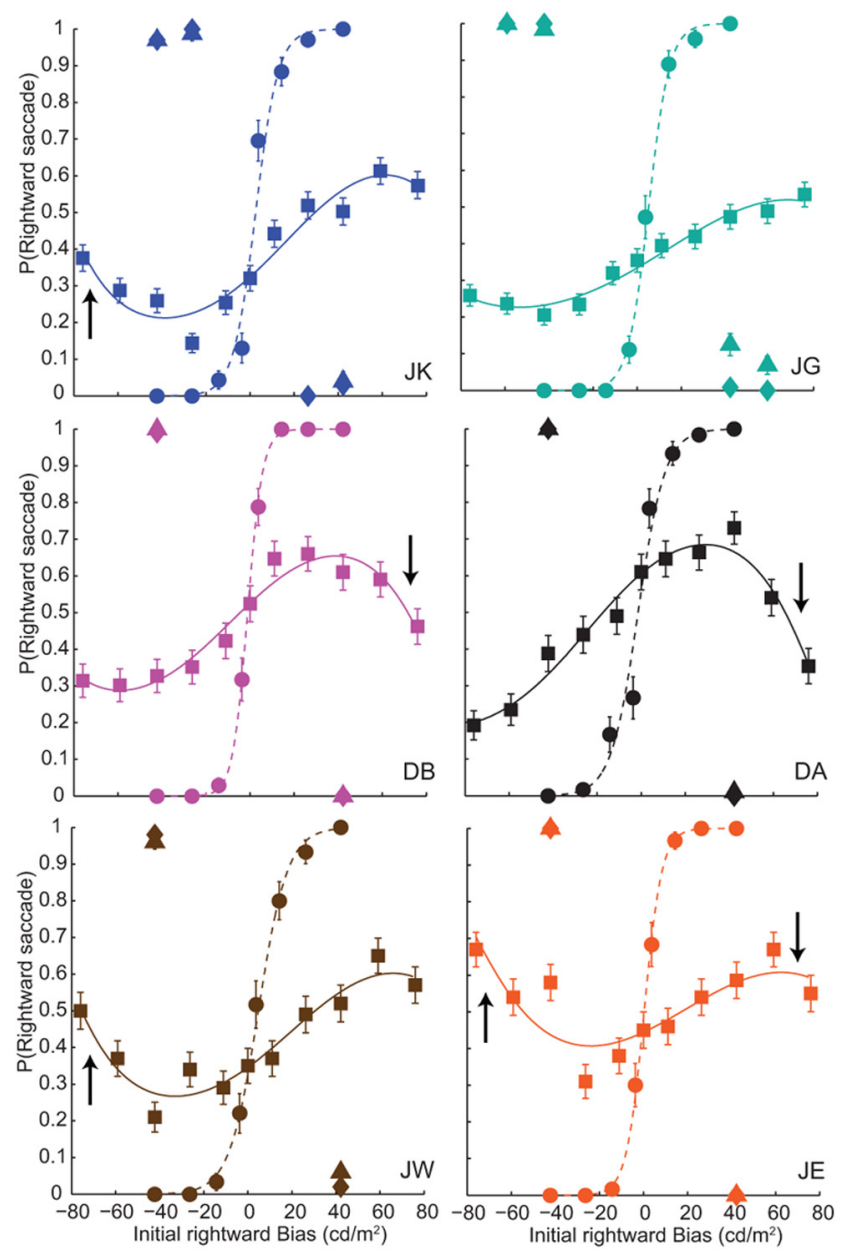

Figure 4. Breakdown of primacy by strong target imbalance and late forcing pulses. Probability of rightward saccades ( \pm SEM) as a function of the (initial) intensity difference between the left and the right target in Experiment 4. Each panel shows the results from one subject. Circles and dotted lines indicate the $50 \mathrm{~ms}$ control condition with a fitted psychometric curve (Equation 1). Squares and solid lines indicate the $50-50 \mathrm{~ms}$ reversal trials ( $n \geq 100$ for each $\Delta /$ ) with fitted curves following Equation 2. Note the nonmonotonic nature of these psychometric response functions. Arrows indicate a significant decrease in the primacy effect (Fischer's exact test, $p<0.05$ ). Forced reversal stimuli induced a near-complete inversion of the primacy effect. Diamonds indicate the 50-50-67 ms forced reversal condition. Triangles indicate the 83-67 forced reversal condition.

thus demonstrate that the observed primacy effects cannot be attributed purely to early choice commitment because, without sufficiently strong feedback competition, the latter would predict a monotonic increase in primacy.

\section{Forced reversal}

Although the basic primacy effect could indeed be reduced by stronger target contrasts (approximately for $|\Delta I|>40 \mathrm{~cd} / \mathrm{m}^{2}$ ), these findings could not provide a strict upper limit on the possible contribution of premature choice commitment or a strict lower limit on the contribution of dynamic hysteresis. To obtain those limits, we investigated to what extent the primacy effect breaks down when later-arriving stimulus evidence in favor of the opposite target location is very strong. Experiment 4 therefore included "forced reversal" trials in which $D_{2}$ was immediately followed by a $D_{3}$ epoch in which the reversed target imbalance was made even stronger than the reversed imbalance during $D_{2}$. As explained in the Materials and Methods and illustrated in Figure $5 A$, any residual probability of choosing the initially 

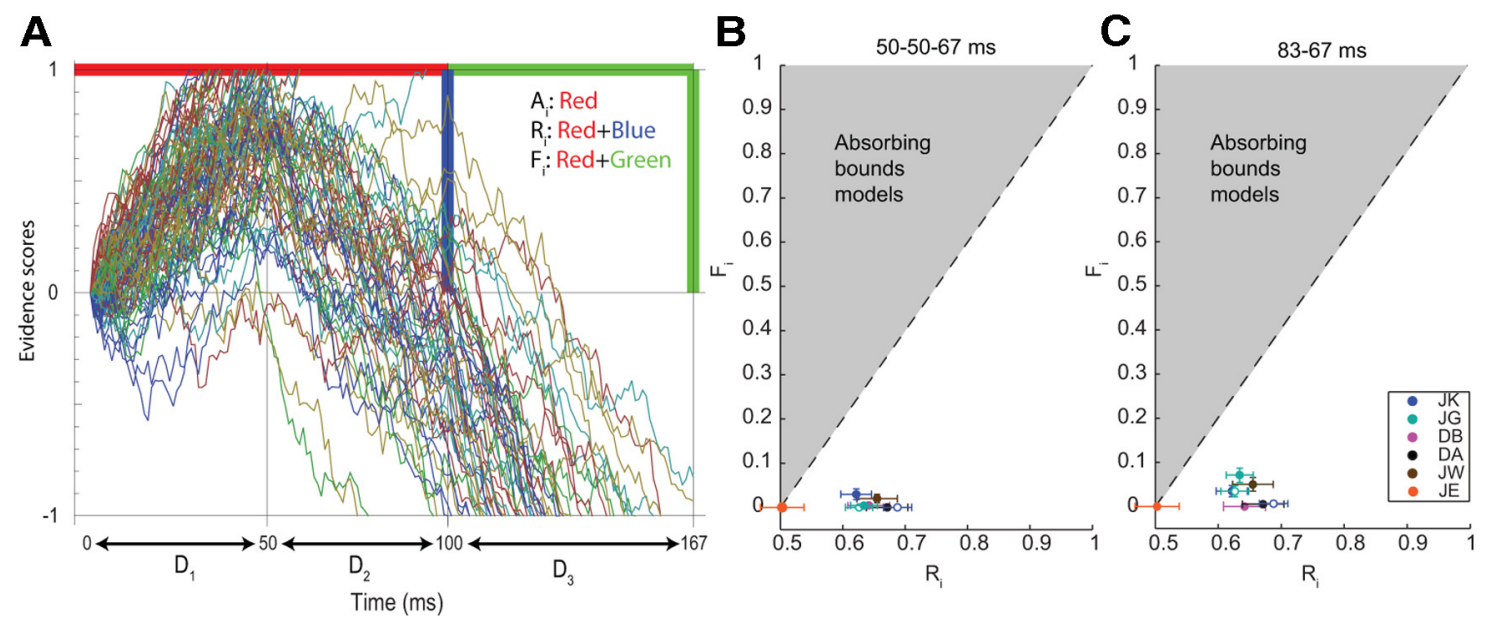

Figure 5. Breakdown of primacy under forced reversal conditions excludes absorbing-bound models. A, Evidence trajectories in the bounded drift diffusion model (Gold and Shadlen, 2007) for the reversal stimuli applied in Experiment 4. The bounded drift diffusion model is an example of a much broader class of decision-making models that all share the common property that the choice for a particular alternative becomes irreversible once the accumulated evidence for that alternative reaches its critical bound (here at either $E=1$ or $E=-1$ ). Three variables are defined: $A_{i,} R_{i,}$ and $F_{i}$. $A_{i}$ is the fraction of trajectories that—according to the model — hit the bound for the initially strongest target in the red-labeled time window. $R_{i}$ is the observed probability of choosing the initially strongest target in the (unforced) reversal condition. $R_{i}$ equals the sum of $A_{i}$ and the fraction of trajectories that crossed the vertical blue line segment. $F_{i}$ is the probability of $s$ till choosing the initially strongest target in the forced reversal condition. $F_{i}$ equals the sum of $A_{i}$ and the fraction of trajectories that hit or crossed the green line segments. Only those trajectories that did not yet hit a bound by the end of $D_{2}$ (i.e., the "surviving" trajectories) can still be influenced by the $D_{3}$ forcing stimulus. $F_{i}$ thus sets the upper limit for $A_{i}$. Together with the antisymmetric nature of the reversal stimulus, this leads to the prediction that, for absorbing bound models, the inequality $F_{i} \geq 2 R_{i}-1$ must hold (Equation 4 , Materials and Methods). $B, C$, Choice probabilities for the initially strongest target in the 50-50-67 ms $(\boldsymbol{B})$ and the 83-67 ms $(\boldsymbol{C})$ forced reversal conditions $\left(F_{i}\right)$ as a function of the probability of making that same choice in the $50-50 \mathrm{~ms}$ (unforced) reversal condition $\left(R_{i}\right)$. Only the gray area, which satisfies the inequality $F_{i} \geq 2 R_{i}-1$ (Equation 4, Materials and Methods), can be reached by absorbing bound models. Colored symbols show mean data points per subject, with an initial bias of 7.2 versus $49.5 \mathrm{~cd} / \mathrm{m}^{2}$ (filled symbols) or 13.7 versus $40.0 \mathrm{~cd} / \mathrm{m}^{2}$ (J.K., blue open symbol) and 3.6 versus $62.7 \mathrm{~cd} / \mathrm{m}^{2}$ (J.G., cyan open symbol). Error bars indicate \pm 1 SEM (see Materials and Methods).

brightest target in this forced reversal condition then sets an upper limit on how much of the primacy seen without the forcing $D_{3}$ phase is caused by premature choice commitment. Conversely, the amount of primacy in the unforced condition that can be attributed to hysteresis is at least as large as the reduction in primacy that occurs when appending the forcing $D_{3}$ phase to the stimulus.

For efficiency, and to ensure that forced reversal trials were relatively rare occurrences in any given block of trials (17\%), we only used a target contrast that produced a strong primacy effect, as measured in pilot experiments. The duration of the forcing $D_{3}$ phase was set at $67 \mathrm{~ms}$. Note that if $D_{3}$ were too short to reach maximal choice forcing, it would merely reduce the test's power to reject the hypothesis of absorbing bounds being responsible for primacy effects. As shown in Figure 4 (isolated diamonds near $p=0$ and $p=1$ ), adding the $D_{3}$ forcing phase to the stimulus completely abolished primacy and drove all subjects to choose almost exclusively the target with final dominance. The same result was obtained with the 83-67 ms forcing condition (Fig. 4, triangles; see Materials and Methods). These findings strongly suggest that the contribution of premature choice commitment was extremely small.

As outlined in the Materials and Methods, the simplest approach to quantifying how strong these data challenge mechanisms that attribute primacy effects to premature commitment is to focus on a slightly more specific class of models with absorbing bounds, namely those without hysteresis in their evidence accumulation. This restriction still includes all "standard" decision models, such as "bounded drift-diffusion" (Gold and Shadlen, 2007). All models that we are aware of that use bounded integration, leaky or not, and independently of their mutual interactions as long as they are too weak to cause hysteresis. This whole class of models can then be tested quantitatively by comparing the probability $F_{i}$ of still choosing the initially strongest target in forced reversal conditions with the probability $R_{i}$, of choosing the initially strongest target in the corresponding unforced reversal conditions. More specifically, we could test whether the measured $R_{i}$ and $F_{i}$ combinations violate the inequality (see Materials and Methods for details): $F_{i} \geq 2 R_{i}-1$. If so, our data would provide strong evidence against the very wide class of models with absorbing bounds and no hysteresis. One can in fact derive less conservative inequalities for the $R_{i}, F_{i}$ combinations when focusing on specific models within this highly diverse class, but this is beyond the scope of the present paper and proved unnecessary for our present purposes.

To visualize the inequality test, we plotted the averaged $R_{i}$ and $F_{i}$ data pairs from each subject (see Materials and Methods) in the scatter plot in Figure 5 (error bars indicate \pm 1 SEM). Note that the data points would have to fall within the gray area delineated by the line $F_{i}=2 R_{i}-1$ to satisfy the predictions of absorbing bound models without hysteresis. It is quite clear, however, that nearly all data from the 50-50-67 ms forcing condition fell well outside of that region (Fig. 5B). A one-sided $z$ test on each data pair (see Materials and Methods) indeed showed a highly significant violation of inequality $4(p<0.00015)$ for all but one of our subjects. When we performed the test with the $F_{i}$ 's derived from the 83-67 ms forcing condition (Fig. 5C), we also found highly significant violations of inequality $4(p<$ 0.00005), except for subject J.E. As can be seen in Figure 4, the lack of statistical significance for J.E. in these tests stems from a single $R_{i}$ outlier data point. If we would have based the test for this subject on his full dataset (i.e., sampling from the fit-function based on all data points), this test too would have strongly violated inequality 4 .

\section{Model simulations}

The choice data from Experiment 4 provide strong evidence against the broad class of accumulator models with absorbing 
A
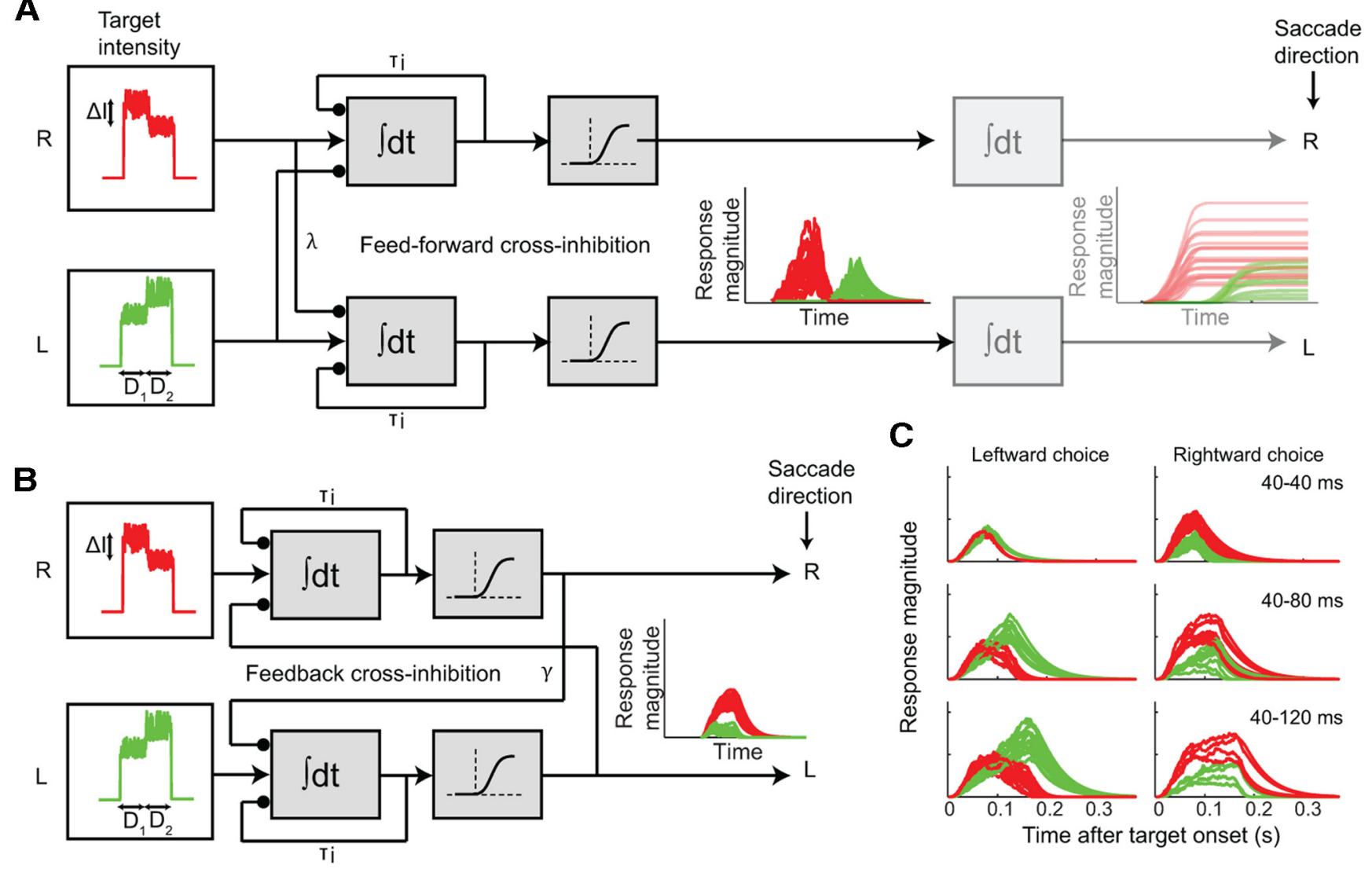

Figure 6. Model properties. $\boldsymbol{A}, \boldsymbol{B}$, Diagram of the feedforward $(\boldsymbol{A})$ and feedback $(\boldsymbol{B})$ cross-inhibition model together with simulations of the $80-80 \mathrm{~ms}$ reversal condition from our experiments. Arrowheads denote excitation, bulletheads inhibition. Inputs represent the intensities of the left $(L, g r e e n)$ and the right $(R$, red) target as a function of time. The inputs pass through a leaky integrator and a nonlinear Naka-Rushton compression stage $\left(s=x^{2} /\left(x^{2}+c\right)\right.$ for $x>0$, otherwise $\left.s=0 ; c=1\right)$. The difference between the two models is in the type of cross-inhibition only. Choices were assigned by comparing the units' response magnitude at stimulus offset. An additional integration step (shown transparently) in the feedforward model can — for this reversal condition — "repair" its failure to produce primacy without absorbing bounds. C, Output of the left (green) and right (red) decision units from the feedback model as a function of time for leftward and rightward choices (columns) in the three different $\left(D_{1}, D_{2}\right)$ timing conditions of Experiment 3 (rows). Model parameters for the simulations in $\boldsymbol{A}-\boldsymbol{C}$, Integration constant $T_{\mathrm{i}}=80 \mathrm{~ms}$; strength of the cross-inhibition, $\gamma=3.33$ for feedback cross-inhibition, $\lambda=1.0$ for feedforward cross-inhibition; Variance of the Gaussian white noise on the inputs was $20 \%$ of the mean.

bounds. Here, we simulated feedforward and feedback competition models without absorbing bounds and tested their performance against our observations. Figure 6 shows a schematic drawing of the feedforward (Fig. 6A) and feedback (Fig. 6B) architectures. The inputs, representing the intensities of the two targets, pass through a leaky-integrator and nonlinear compression stage. Decisions are based on the units' activity levels at target offset. The two models differ only in the type of crossinhibition that mediates the competition. In the feedforward model, each decision unit receives cross-inhibition from the opposing input, whereas in the feedback model, each unit receives cross-inhibition from the output of the alternate decision unit. To show the effect of changes in stimulus intensity, we simulated reversal trials. For the feedback circuit, the nature of the response (degree of hysteresis) depends crucially on how $D_{1}$ relates to the time constant of the overall feedback dynamics. For our first demonstration (Fig. 6B), we simulated the $80-80 \mathrm{~ms}$ reversal condition. The time constant of the decision units was chosen as just sufficient to allow the network to settle on a choice determined by the initially strongest target before the beginning of $D_{2}$ (i.e., $T_{\mathrm{i}}=$ $80 \mathrm{~ms}$ ). This prevents the decision unit of the initially weaker target from responding significantly despite the fact that the target intensities have reversed (Fig. $6 \mathrm{~B}$, insets). Conversely, the output of the feedforward model more or less follows the sequence of input events (Fig. $6 \mathrm{~A}$, insets). Figure $6 \mathrm{C}$ illustrates how the behavior of the feedback model changes if $D_{1}$ is shorter than the feedback time constant. Increasing $D_{2}$ then results in a transition from a preference for the initial brightest target if $D_{2}$ is short (top row) to a preference for the most intense target at the end if $D_{2}$ becomes large (bottom rows).

Figure 7 shows the choice behavior of the two models (same format as Figs. 2, 3). Five different conditions were simulated. The first three panels in each row show simulations of the reversal conditions from Experiment 2 (from left to right, 40-120 ms, 80-80 ms and 120-40 ms, respectively). The fourth panel shows the 40-40 ms reversal condition from Experiment 1. The last panel on the right shows the 50-50 ms reversal condition that was tested over a larger contrast range in Experiment 4. The two curves in each graph show the results obtained with two different parameter sets, representing two subjects that showed distinctly different choice behavior in the 40-120 ms reversal condition of Experiment 2 (gray: D.A.-like subject; black: J.K.-like subject).

The feedback model (Fig. 7A) generally prefers the initially most intense target. However, in the 40-120 ms reversal condition (Fig. $7 \mathrm{~A}$, left), the presence of hysteresis depends critically on the integration constant $\left(T_{\mathrm{i}}\right)$ and the cross-inhibition strength $(\gamma)$. With a longer $T_{\mathrm{i}}$ and a weaker $\gamma$, the hysteresis disappears (note that the absolute values of $T_{\mathrm{i}}$ should not be taken literally because they scale with the shape parameter $c$ of the sigmoid function). For high target contrasts (Fig. 7A, right), the model 


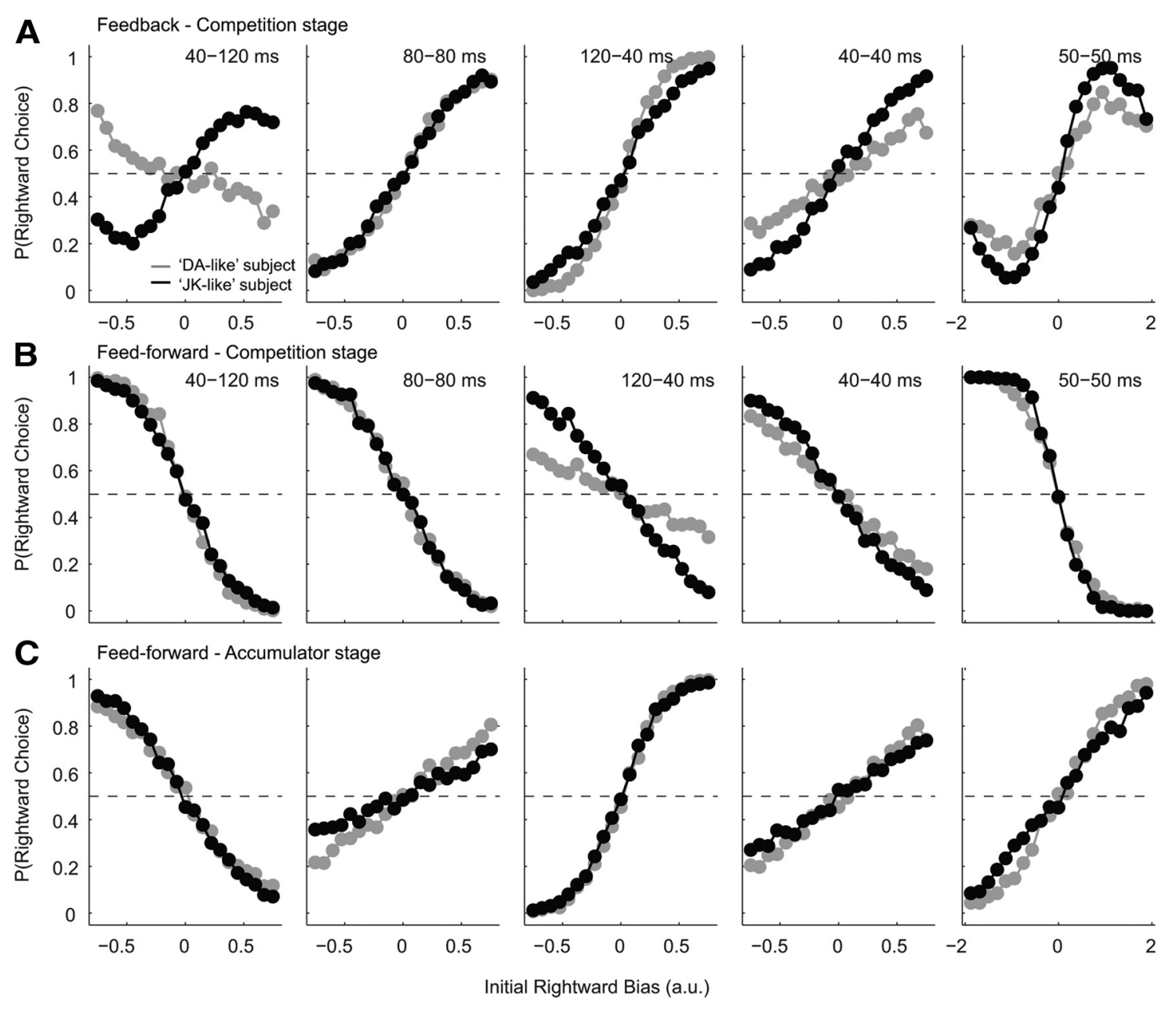

Figure 7. Model simulations of the different reversal conditions. $\boldsymbol{A}$, Choice behavior of the feedback model. $\boldsymbol{B}, \boldsymbol{C}$, Choice behavior of the feedforward model in the competition stage $(\boldsymbol{B})$ and the accumulator stage $(\boldsymbol{C})$ presented in the same format. Each panel shows the probability of choosing the right target as a function of rightward bias at stimulus onset $(\Delta /$, here in arbitrary units) for different timing conditions, as indicated in the top right corner. Note the larger range of $\Delta /$ values on the abscissa of the rightmost panels, corresponding to the larger contrast-range that was tested in Experiment 4. Black and gray symbols represent simulation results obtained with two different sets of parameters. Values in each set were chosen to either mimic the behavior of subject D.A. (gray) or subject J.K. (black), because these two subjects clearly showed opposite choice behavior in the 40-120 ms reversal condition of Experiments 2 and 3 . Model parameters were obtained by manual adjustment: $T_{\mathrm{i}}=50 \mathrm{~ms}$ and $T_{\mathrm{i}}=25 \mathrm{~ms}$ (gray and black curves, respectively); $\gamma=3.25$ and $\gamma=7.00$ for feedback cross-inhibition (gray and black curves, respectively), $\lambda=1$ for feedforward cross-inhibition; Variance of the Gaussian white noise on the inputs was $60 \%$ of the mean. Each data point consists of 500 simulated trials.

shows the observed decline of the primacy effect as such strong stimuli are able to "override" the hysteresis. Response biases as observed in the experiments could be induced, for example, by asymmetries in input gains for the left and right decision units (data not shown).

For the feedforward model, a direct readout of the competition stage at stimulus offset (Fig. $7 B$ ) always predicts a preference for the target that is most intense at the end. Note, however, that this failure to replicate the basic primacy effect can be "repaired" by adding an accumulator stage that integrates the output of the competition stage without any further interactions or absorbing bounds (Fig. 6A). Indeed, when the choices are based on the total time integral of its competition stage output (Fig. 7C), primacy occurs when the two stimulus epochs are equal or the first epoch is longer. Nevertheless, with this augmented version of the feedforward model, it remains impossible to produce primacy in the 40-120 ms reversal condition (as seen in some of our subjects in Experiments 2 and 3). This is due to the absence of absorbing bounds and absence of hysteresis in the evidence accumulation. Both versions of the feedforward model also fail to account for the observed decline in primacy at large target contrasts (Exper- iment 4) regardless of its parameter settings. Thus, in short, neither the competition stage nor the added accumulator stage of the feedforward model were able to fully capture all key features of the saccadic choice behavior that were revealed across our different reversal conditions.

\section{Reaction time}

The choice patterns in Experiments 2 and 3 showed remarkably different dependencies on the same stimulus set between our subjects. This offers a unique opportunity to investigate whether reaction times for the alternatives follow similarly different patterns in the same subjects or if they are dissociated from choice probabilities altogether. We wondered, for example, if the relation between reaction time and choice probability followed some simple constraints such as: (1) the mean latencies of saccades toward the most frequently chosen target are systematically shorter than the mean latencies of saccades toward the competing target, and (2) increases in choice preference are associated with increases in this latency difference.

The data from Experiments 1-3 showed, however, that there was no significant correlation between choice preference and 
mean latency difference of leftward versus rightward saccades in the control conditions (Fig. $8 A ; r=0.11, t$ test, $p=0.28$ over all subjects and conditions). For most data from the reversal conditions (Fig. 8B), we did find a significant correlation between choice preference and latency difference $(r=0.65, t$ test, $p \ll$ 0.0001 ), but only for timing conditions that produced a preference for the initially brightest target (Fig. 8B, small symbols, thin regression line). For timing conditions that produced a preference for the target with the highest final intensity (i.e., negative slopes of psychometric curves, $\sim 20 \%$ of the data), we actually found an opposite relationship between latency and choice. Under these latter conditions (Fig. $8 B$, large symbols, thick regression line), the mean latencies of rightward saccades increased significantly compared with the mean latencies of leftward saccades as the preference for the right target increased and vice versa if preference for the left target increased $(r=-0.68, t$ test, $p \ll$ 0.0001 ).

This remarkable dissociation between choice probability and saccade latency is further illustrated in Figure $8 C-E$ for the four subjects that showed nearly inverted choice preferences in the $80-80 \mathrm{~ms}$ (black) versus $40-120 \mathrm{~ms}$ (gray) reversal conditions in Experiment 2 (Fig. $3 B, D$ ). Note that for each saccade direction, the changes in reaction time as a function of initial target contrast were the same under both timing conditions (Fig. $8 D, E$; ANCOVA, $F=0.24, p>0.8$ and $F=1.88, p>0.1$, respectively), whereas the changes in choice probability were opposite (Fig. $8 C$, $t$ test, $p<0.0001$ ). This shows that the reaction times depended strongly on the initial target contrast and on the direction of the ensuing saccade, but not on the probability of choosing either target. Saccade latency was short compared with the ambiguous control condition if the initially stronger target was chosen (i.e., $\Delta R T<0$ ) but long if the initially weaker target was chosen (i.e., $\Delta R T>0$ ), and these latency differences increased as a function of the initial intensity difference regardless of the choice probability.

This relation between reaction time and the initial intensity of the selected target (or its initial intensity difference with the other target) was found across all experiments and all conditions (Fig. 9). In Experiments 1-3, the chronometric response functions obtained in reversal conditions (Fig. $9 B$ ) were actually quite similar to the ones obtained in control conditions (Fig. 9A). This is quantified in Figure $9 C$ for all test conditions applied in Experiment 1 using the slopes of the chronometric response functions (see Materials and Methods, above; Eq. 8). Because the chronometric functions for rightward and leftward saccades were practically mirror images, we pooled these responses by inverting the slope of the rightward responses. Neither the $80-80 \mathrm{~ms}$ nor the 40-40 ms reversal condition produced significantly different latency effects compared with the control conditions (ANCOVA, $F=1.18, p>0.2$ and $F=0.5, p>0.4$, respectively). Similar results were obtained in Experiments 2 and 3 (Fig. 9D, E, respec- tively). In both experiments, changes in $D_{1}$ and $D_{2}$ had very little influence on the saccade latencies. This contrasts markedly with the robust influences on choice behavior (Fig. 3). In fact, the chronometric functions remained invariant to both the presence and timing of the reversals (ANCOVA: no significant differences in Experiment 2, $F=0.28, p>0.8$ and Experiment $3, F=1.25, p>0.2)$. This similarity between control and reversal data in Experiments 1-3 shows that a revision of the initial decision by later evidence did not lead to an extra delay in saccade reaction time. In fact, in Experiment 4, when we forced saccades in the opposite direction of the initially strongest target, thus breaking down the hysteresis built up during the initial part of the stimulus, the latencies were somewhat shorter than the ones in the corresponding unforced condition (mean \pm SEM latency difference for movements to the same location: $26 \pm 4 \mathrm{~ms}$, paired $t$ test, $p<0.0001$; and for movements to the opposite location: $17 \pm 5 \mathrm{~ms}, p<0.01$; data not shown). This latency reduction by late stimulus evidence also refutes the notion that initial information alone might have been the determining factor in reaction times. The latter is further corroborated by the significant differences in slopes of the chronometric function for the control versus reversal conditions obtained in Experiment 4 (Fig. 9F; ANCOVA: $F=$ $47.53, p<0.0001$ ), for which the mean target intensity levels were larger than in Experiments 1-3 (i.e., $27.8 \mathrm{~cd} / \mathrm{m}^{2}$ vs 16.7 $\mathrm{cd} / \mathrm{m}^{2}$, respectively; see Materials and Methods). 

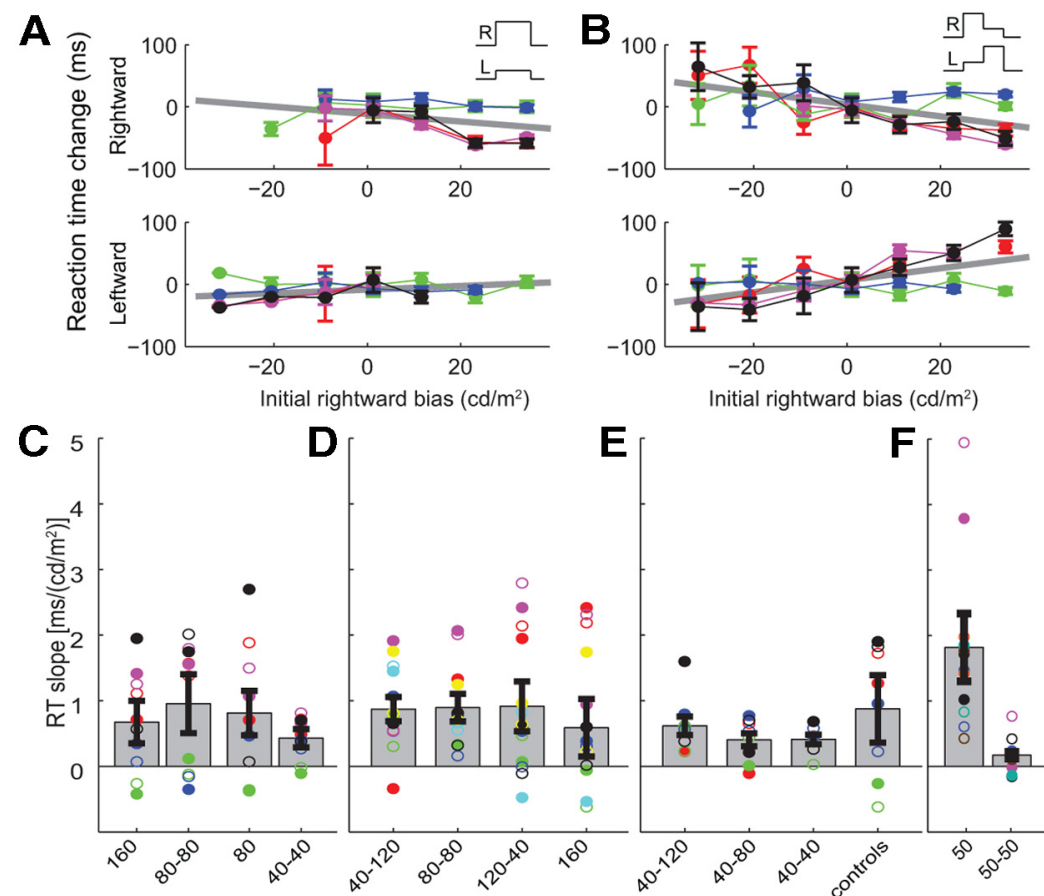

D

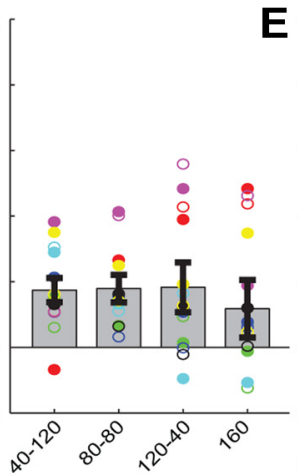

Condition (ms)

Figure 9. Reaction time as a function of initial intensity of the selected target. $A, B$, Average reaction times relative to stimulus onset as a function of the initial rightward bias in the $160 \mathrm{~ms}$ control condition $(\boldsymbol{A})$ and the $80-80 \mathrm{~ms}$ reversal condition $(\boldsymbol{B})$ of Experiment 1. Averages ( \pm 1 SEM) were calculated from a minimum of six responses per intensity condition (i.e., for choice probabilities, $p \geq 0.1$ ). For each subject, the average reaction time measured in the ambiguous condition was subtracted before averaging to correct for large intersubject variation in average reaction time and the results were shown separately for saccades to the right (top) and the left (bottom) target. As a result, offsets of the regression lines (gray) were practically zero. Colored lines and symbols are results from individual subjects. Thick regression lines (gray) are based on the pooled data from all subjects. Average reaction times $( \pm S D)$ in the ambiguous condition for the different subjects ranged between $395 \pm 15 \mathrm{~ms}$ and $573 \pm 11 \mathrm{~ms}$. $\boldsymbol{C}-\boldsymbol{F}$, Slopes of the chronometric functions (i.e., gray lines in $\boldsymbol{A}, \boldsymbol{B}$ ) for the different experimental conditions of Experiments $1-4$, respectively. Colored symbols represent slopes from individual subjects. To allow for pooling of the data from leftward (open symbols) and rightward saccades (filled symbols), slopes obtained for rightward responses were multiplied by -1 . The resulting slopes were typically positive, indicating that reaction times decreased with increasing initial intensity of the selected target. Bars with error bars show the mean \pm 1 SEM across subjects.

\section{Discussion}

We studied saccadic decision making using two simultaneously ( $\leq 160 \mathrm{~ms}$ ) presented targets with intensity reversals occurring at different moments in time. Using this novel approach, we found a robust primacy effect: subjects always preferred the target that was brightest during the early part of each stimulus even if longerlasting, opposite differences were present in the second part of the stimulus. This primacy effect collapsed when: (1) the duration of the early stimulus phase was reduced to $\sim 40 \mathrm{~ms}$, (2) the target intensity differences were large, or (3) the reversal stimulus was followed by a strong stimulus bias toward the initially weakest target. The latter two findings show that the basic primacy effect did not result from premature commitment to the initially dominant target. A decision model that assumes feedback crossinhibition, however, fully described the observed choice behavior.

There is some prior evidence that decision making is biased toward early visual information. In a motion discrimination task, Ludwig et al. (2005) found that subjects rely mostly on information provided in the beginning, a finding that they later explained with a time-varying decision threshold (Ludwig, 2009). Disturbing motion pulses also have more effect when they occur early in a trial (Huk and Shadlen, 2005; Kiani et al., 2008; Tsetsos et al., 2012). Furthermore, using a search task in which target intensities jittered, Tsetsos et al. (2011) found a preference for the initially strongest target and concluded that this could be due to feedback competition.

One might argue that feedforward models do predict primacy if subjects adopted a low decision threshold reached shortly after stimulus onset (Ludwig, 2009) or if the integration of evidence were bounded (Kiani et al., 2008). However, our finding that the primacy effect decreases at higher target contrasts (Fig. 4) cannot be explained by any feedforward model with irreversible decision thresholds. Moreover, the very low probabilities of still choosing the initially strongest target in the forced reversal condition (Fig. 5) show that even for feedback models that do allow dynamic hysteresis, absorbing bounds play no significant role in controlling the well defined saccadic choice behavior that occurred in Experiment 4. These robust findings are corroborated by some of the results obtained by Tsetsos et al. (2012), who found that motiondirection discrimination in one of their subjects showed a primacy-to-recency transition for short- versus long-duration stimuli. Given the evidence against choice commitment, dominance of the initial stimulus also contradicts many other model types: high-leakage models (Kiani et al., 2008) and most "urgency" models (Ditterich, 2006; Cisek et al., 2009) give more weight to the final stimulus (unless the urgency signal rose faster than our $40 \mathrm{~ms}$ minimal duration; Standage et al., 2011). Likewise, recent gating models (Purcell et al., 2010; Schall et al., 2011) prevent accumulation of the initial, low-amplitude part of the sensory input.

In theory, it is possible that a different mechanism triggered a saccade to the bright forcing target even though the original mechanism had already committed to the other target. In particular, one might worry that, due to subsiding fixation activity, the late forcing pulses were able to elicit so-called express saccades (Dorris et al., 1997; Bell et al., 2006). There was, however, no evidence for this; the latencies of individual saccades were always $>150 \mathrm{~ms}$ relative to the onset of the forcing pulse, which rules out any specific involvement of the express pathways. We also examined the actual eye movement traces. Prior commitment to the initially strongest target in a parallel pathway would predict that saccades toward the forcing target are substantially influenced by (preparatory) activity related to the other impending saccade. However, as shown in Figure 10, the metrics and kinematics of saccades in the forced condition were actually very similar to those in the control and unforced condition. This supports our assumption that they resulted from the same saccade mechanism.

Interestingly, we found that reaction times depended on the initial stimulus contrast and on the direction of the ensuing saccade (Fig. 9), but not on the probability of choosing either target (Fig. 8). In fact, by manipulating the timing of the intensity swaps in Experiments 2 and 3, we could nearly invert the relation between reaction time and choice probability from faster to slower 
A Control condition
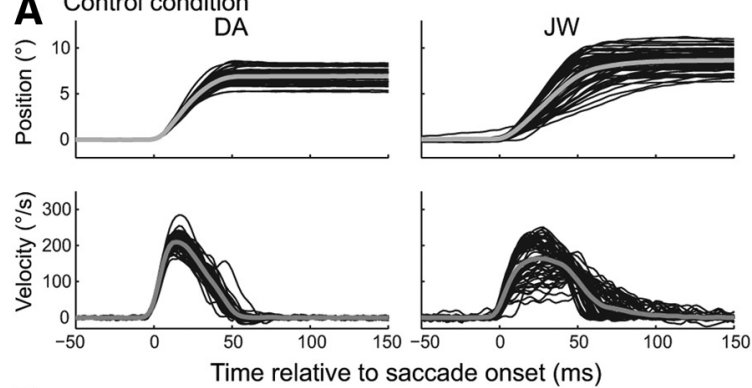

B Reversal condition
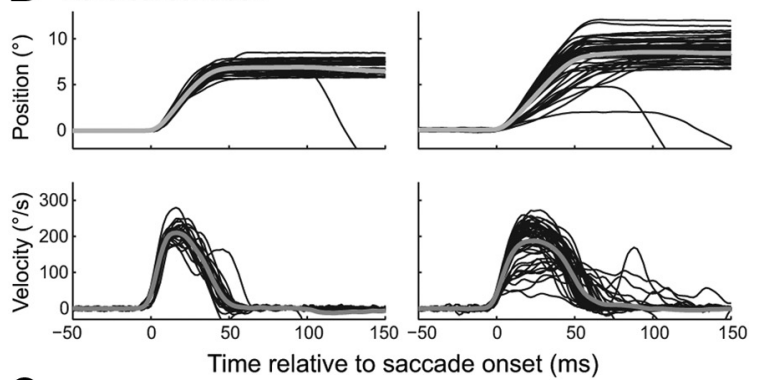

C Forced reversal condition
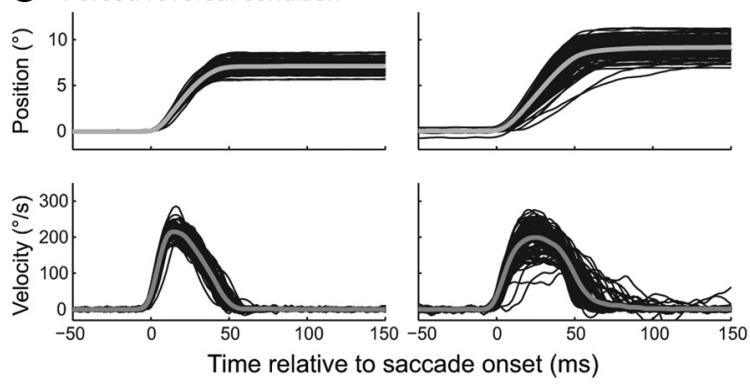

Figure 10. Saccade trajectories. Position and velocity traces of all saccades to the left target in the control $(\boldsymbol{A})$, reversal $(\boldsymbol{B})$, and forced reversal $(\boldsymbol{C})$ condition made by two naive subjects (D.A. and J.W.) in Experiment 4 (black). Averaged traces are superimposed (gray). Both the metrics and kinematics of the saccades were comparable. This supports our assumption that they resulted from the same saccade mechanism.

reactions for the more likely outcome in some of our subjects. Such a remarkable dissociation between reaction time and choice behavior has, to our knowledge, never been reported. Instead, it is typically found that changes in choice probability go hand in hand with opposite changes in reaction time (Palmer et al., 2005; Chittka et al., 2009, but see Niwa and Ditterich, 2008, who found another example of a dissociation: reaction times that varied across conditions without changes in choice probability and vice versa). Current decision-making theories explain these findings from the first-to-threshold principle, but this principle cannot account for the choice behaviors observed in Experiment 4 (Figs. 4,5 ), the decoupling of choices and reaction times (Fig. 8), and the remarkable invariance of the chronometric functions to both the presence and timing of the reversals observed in Experiments 1-3 (Fig. 9A-E). We thus conclude that reaction time and choice are determined by separate mechanism rather than by a singlestage competition process. This fits in previous conceptual schemes (Findlay and Walker, 1999), which propose that "when" and "where" are determined by parallel but hierarchically organized pathways.

Physiological studies clearly support the notion that target selection and movement initiation are distinct phenomena. In monkeys performing visual search, visually responsive cells in frontal eye fields, lateral intraparietal sulcus, and superior collicu- lus discriminate between target and distracters (frontal eye field: Schall and Hanes, 1993; lateral intraparietal sulcus: Schall and Hanes, 1993; Ipata et al., 2006; Thomas and Paré, 2007; superior colliculus: Basso and Wurtz, 1997; McPeek and Keller, 2002), but the latency with which these cells discriminate the target from distractors is unrelated to the timing of the ensuing movement. In fact, the selection takes place independently of movement execution (Schall et al., 1995; Thompson et al., 1996; Thompson et al., 1997; Murthy et al., 2001; Sato and Schall, 2003; Juan et al., 2004; Murthy et al., 2009).

The chronometric functions from all of our experiments indicated that reaction times decreased systematically with increasing initial intensity of the selected target (Figs. 8, 9). One might speculate, therefore, that the reaction times might have been determined by the target contrasts at stimulus onset. Such a theory would be consistent, for example, with the contrast-dependent spike timing recently observed in primary visual cortex (Lee et al., 2010) and is further supported by Experiments 1-3, which showed that neither the presence nor the timing of the intensity reversals had a significant influence on the chronometric response curves (Fig. 9C-E), However, in Experiment 4, we found that the chronometric functions for the control and reversal conditions were clearly different (Fig. $9 F$ ) and that the later forcing stimulus actually shortened the saccade latencies, indicating that later stimulus information did affect the reaction times. A possible interpretation of these effects is that increases in stimulus intensity decrease the delays in visual processing (Bell et al., 2006).

The present literature leaves some uncertainty about how the target-selection stage is read out. In our simulations, we tested two different approaches. Either the integrated output of the decision units was compared, choosing the channel with the highest value as the current winner, or the output levels of the decision units at stimulus offset were compared. The latter could rely, for example, on the activity levels of cells that keep the latest choice in working memory (Kojima and Goldman-Rakic, 1982; Chafee and Goldman-Rakic, 1998). For the feedforward model, the type of readout heavily influenced its choice behavior, but due to the absence of hysteresis in the evidence accumulation, neither type could be reconciled with the experimental data (Fig. 7 B, C). For the feedback model, only a readout at stimulus offset produced predictions that were qualitatively consistent with the observed behavior (Fig. 7A). Results from the integrated output (data not shown) failed to account for the nonmonotonic nature of the psychometric curves in the 50-50 ms reversal conditions of Experiment 4.

Bollimunta and Ditterich (2012) obtained physiological evidence from monkey lateral intraparietal sulcus for the presence of a feedforward inhibition component in the random-dot motiondirection discrimination task. At the same time, the presence of feedback inhibition could not be ruled out. Here, we did not simulate hybrid models. It is possible, and perhaps even likely, that feedforward inhibition also contributes to the visual targetselection process that we have studied here. However, having ruled out a significant contribution of premature choice commitment in Experiment 4, the essence of our findings is that the observed primacy effects can only be accounted for by a model that includes sufficiently strong feedback cross-inhibition.

We conclude that saccadic responses to competing visual targets are best described by a model featuring a competitive choice mechanism based on feedback cross-inhibition that exerts executive control (possibly mediated by the substantia nigra; Hikosaka and Wurtz, 1983) over the initiation of upcoming saccades. 


\section{References}

Basso MA, Wurtz RH (1997) Modulation of neuronal activity by target uncertainty. Nature 389:66-69. CrossRef Medline

Bell AH, Meredith MA, Van Opstal AJ, Munoz DP (2006) Stimulus intensity modifies saccadic reaction time and visual response latency in the superior colliculus. Exp Brain Res 174:53-59. CrossRef Medline

Bogacz R, Brown E, Moehlis J, Holmes P, Cohen JD (2006) The physics of optimal decision making: a formal analysis of models of performance in two-alternative forced-choice tasks. Psychol Rev 113:700-765. CrossRef Medline

Bollimunta A, Ditterich J (2012) Local computation of decision-relevant net sensory evidence in parietal cortex. Cereb Cortex 22:903-917. CrossRef Medline

Chafee MV, Goldman-Rakic PS (1998) Matching patterns of activity in primate prefrontal area $8 \mathrm{a}$ and parietal area 7ip neurons during a spatial working memory task. J Neurophysiol 79:2919-2940. Medline

Chittka L, Skorupski P, Raine NE (2009) Speed-accuracy tradeoffs in animal decision making. Trends Ecol Evol 24:400-407. CrossRef Medline

Cisek P, Puskas GA, El-Murr S (2009) Decisions in changing conditions: the urgency-gating model. J Neurosci 29:11560-11571. CrossRef Medline

Cousineau D (2004) Merging race models and adaptive networks: a parallel race network. Psychon Bull Rev 11:807-825. CrossRef Medline

Ditterich J (2006) Evidence for time-variant decision making. Eur J Neurosci 24:3628-3641. CrossRef Medline

Dorris MC, Paré M, Munoz DP (1997) Neuronal activity in monkey superior colliculus related to the initiation of saccadic eye movements. J Neurosci 17:8566-8579. Medline

Findlay JM, Walker R (1999) A model of saccade generation based on parallel processing and competitive inhibition. Behav Brain Sci 22:661-721. Medline

Furman M, Wang XJ (2008) Similarity effect and optimal control of multiple-choice decision making. Neuron 60:1153-1168. CrossRef Medline

Gold JI, Shadlen MN (2007) The Neural Basis of Decision Making. Annu Rev Neurosci 30:535-574. CrossRef Medline

Hikosaka O, Wurtz RH (1983) Visual and oculomotor functions of monkey substantia nigra pars reticulata. IV. Relation of substantia nigra to superior colliculus. J Neurophysiol 49:1285-1301. Medline

Huk AC, Shadlen MN (2005) Neural activity in macaque parietal cortex reflects temporal integration of visual motion signals during perceptual decision making. J Neurosci 25:10420-10436. CrossRef Medline

Ipata AE, Gee AL, Goldberg ME, Bisley JW (2006) Activity in the lateral intraparietal area predicts the goal and latency of saccades in a freeviewing visual search task. J Neurosci 26:3656-3661. CrossRef Medline

Juan CH, Shorter-Jacobi SM, Schall JD (2004) Dissociation of spatial attention and saccade preparation. Proc Natl Acad Sci U S A 101:15541-15544. CrossRef Medline

Kiani R, Hanks TD, Shadlen MN (2008) Bounded integration in parietal cortex underlies decisions even when viewing duration is dictated by the environment. J Neurosci 28:3017-3029. CrossRef Medline

Kojima S, Goldman-Rakic PS (1982) Delay-related activity of prefrontal neurons in rhesus monkeys performing delayed response. Brain Res 248: 43-49. CrossRef Medline

Laming DR (1966) A new interpretation of the relation between choicereaction time and the number of equiprobable alternatives. Br J Math Stat Psychol 19:139-149. CrossRef Medline

Lee J, Kim HR, Lee C (2010) Trial-to-trial variability of spike response of V1 and saccadic response time. J Neurophysiol 104:2556-2572. CrossRef Medline

Link SW, Heath RA (1975) A sequential theory of psychological discrimination. Psychometrika 40:77-105. CrossRef

Ludwig CJ (2009) Temporal integration of sensory evidence for saccade target selection. Vis Res 49:2764-2873. CrossRef Medline

Ludwig CJ, Gilchrist ID, McSorley E, Baddeley RJ (2005) The temporal impulse response underlying saccadic decisions. J Neurosci 25:9907-9912. CrossRef Medline

McPeek RM, Keller EL (2002) Saccade target selection in the superior colliculus during a visual search task. J Neurophysiol 88:2019-2034. Medline Murthy A, Thompson KG, Schall JD (2001) Dynamic dissociation of visual selection from saccade programming in frontal eye field. J Neurophysiol 86:2634-2637. Medline

Murthy A, Ray S, Shorter SM, Schall JD, Thompson KG (2009) Neural control of visual search by frontal eye field: effects of unexpected target displacement on visual selection and saccade preparation. J Neurophysiol 101:2485-2506. CrossRef Medline

Niwa M, Ditterich J (2008) Perceptual decisions between multiple directions of visual motion. J Neurosci 28:4435-4445. CrossRef Medline

Noest AJ, van Ee R, Nijs MM, van Wezel RJA (2007) Percept-choice sequences driven by interrupted ambiguous stimuli: a low-level neural model. J Vis 7:1-14. CrossRef Medline

Nosofsky RM, Palmeri TJ (1997) An exemplar-based random walk model of speeded classification. Psychol Rev 104:266-300. CrossRef Medline

Palmer J, Huk AC, Shadlen MN (2005) The effect of stimulus strength on the speed and accuracy of a perceptual decision. J Vis 5:376-404. CrossRef Medline

Palmeri TJ (1997) Exemplar similarity and the development of automaticity. J Exp Psychol Learn Mem Cogn 23:324-354. CrossRef Medline

Purcell BA, Heitz RP, Cohen JY, Schall JD, Logan GD, Palmeri TJ (2010) Neurally constrained modeling of perceptual decision making. Psychol Rev 117:1113-1143. CrossRef Medline

Ratcliff R, McKoon G (2008) The diffusion decision model: theory and data for two-choice decision tasks. Neural Computation 20:873-922. CrossRef Medline

Sato TR, Schall JD (2003) Effects of stimulus-response compatibility on neural selection in frontal eye field. Neuron 38:637-648. CrossRef Medline

Schall JD, Hanes DP (1993) Neural basis of saccade target selection in frontal eye field during visual search. Nature 366:467-469. CrossRef Medline

Schall JD, Hanes DP, Thompson KG, King DJ (1995) Saccade target selection in frontal eye field of macaque. I. Visual and premovement activation. J Neurosci 15:6905-6918. Medline

Schall JD, Purcell BA, Heitz RP, Logan GD, Palmeri TJ (2011) Neural mechanisms of saccade target selection: gated accumulator model of the visualmotor cascade. Eur J Neurosci 33:1991-2002. CrossRef Medline

Smith PL, Ratcliff R (2004) Psychology and neurobiology of simple decisions. Trends Neurosci 27:161-168. CrossRef Medline

Sprenger A, Neppert B, Köster S, Gais S, Kömpf D, Helmchen C, Kimmig H (2008) Long-term eye movement recordings with a scleral search coileyelid protection device allows new applications. J Neurosci Methods 170:305-309. CrossRef Medline

Standage D, You H, Wang DH, Dorris MC (2011) Gain modulation by an urgency signal controls the speed-accuracy trade-off in a network model of a cortical decision circuit. Front Comput Neurosci 5:7. CrossRef Medline

Thomas NW, Paré M (2007) Temporal processing of saccade targets in parietal cortex area LIP during visual search. J Neurophysiol 97:942-947. CrossRef Medline

Thompson KG, Hanes DP, Bichot NP, Schall JD (1996) Perceptual and motor processing stages identified in the activity of macaque frontal eye field neurons during visual search. J Neurophysiol 76:4040-4055. Medline

Thompson KG, Bichot NP, Schall JD (1997) Dissociation of visual discrimination from saccade programming in macaque frontal eye field. J Neurophysiol 77:1046-1050. Medline

Tsetsos K, Usher M, McClelland JL (2011) Testing multi-alternative decision models with non-stationary evidence. Front Neurosci 5:63. Medline

Tsetsos K, Gao J, McClelland JL, Usher M (2012) Using time-varying evidence to test models of decision dynamics: bounded diffusion vs. the leaky competing accumulator model. Front Neurosci 6:79. CrossRef Medline

Usher M, McClelland JL (2001) The time course of perceptual choice: the leaky, competing accumulator model. Psychol Rev 108:550-592. CrossRef Medline

van Zandt T, Ratcliff R (1995) Statistical mimicking of reaction time data: Single-process models, parameter variability, and mixtures. Psychonomic Bulletin and Review 2:20-54. CrossRef

Wang XJ (2002) Probabilistic decision making by slow reverberation in cortical circuits. Neuron 36:955-968. CrossRef Medline

Wong KF, Wang XJ (2006) A recurrent network mechanism of time integration in perceptual decisions. J Neurosci 26:1314-1328. CrossRef Medline 